\title{
A MINI-REVIEW ON SKEETER SYNDROME OR LARGE LOCAL ALLERGY TO MOSQUITO BITES \\ By
}

AMR M. EL-SAYED ABDEL-MOTAGALY ${ }^{1}$, HANAA MAHMOUD MOHAMAD ${ }^{1}$ and TOSSON A. MORSY ${ }^{2}$

Military Medical Academy ${ }^{1}$, Cairo 11291 and Department of Parasitology, Faculty of Medicine, Ain Shams University², Cairo 11566, Egypt

Abstract

Skeeter Syndrome is an allergy to mosquito saliva secreted while taken a human blood meal. It is present with extreme swelling, itching, blistering, infection, fever and general malaise, some cases develop asthma and cellulitis and even threatening anaphylactic shock. Most people of all ages particularly small children, toddlers and seniors who suffer from skeeter syndrome experience a very extreme reaction showed some allergic reaction level, with itching and redness. Sometimes, the swelling is painful and so extreme that the affected limb doubles in size, eyes swell shut, and the area feels hot and hard to the touch or the bite will blister and ooze.

Key words: Mosquito bite, Skeeter syndrome, Differential diagnosis, Treatment, Prevention.

\section{Introduction}

The reactions to mosquito bites are caused by an immunologic response to proteins (polypeptides) in mosquito saliva. Many people who are bitten by mosquitoes develop an immune response to these proteins; however, only a small proportion of them develop clinically relevant allergic reactions, most commonly large local reactions (Peng and Simons, 2007).

Types of reactions due to mosquito bites: There are two main types of mosquito bites 1- Typical (normal) reactions: Typical local cutaneous reactions to mosquito bites consist of immediate wheals (swelling) with surrounding flares (redness) peaking at 20 minutes, and delayed itchy, indurated (firm) papules peaking at 24 to 36 hours, which diminish over 7 to 10 days.

The typical clinical course of sensitization and natural desensitization to the salivary proteins injected when mosquitoes bite was described initially in the 1940s. It evolves over months or years. People who have never been exposed to a particular species of mosquito do not develop reactions to the initial bites from such mosquitoes. Subsequent bites result in the appearance of delayed local skin reactions. After repeated bites, immediate wheals develop (Mellanby, 1946). With further exposure, the delayed local reactions wane and eventually disappear, alt- hough the immediate reactions persist. People who are repeatedly exposed to bites from the same species of mosquito eventually also lose their immediate reactions. The duration of each of these five different stages differs, depending on the intensity and timing of mosquito exposure (Reunala et al, 1994). These typical reactions are annoying, but should not cause undue alarm. The immunological basis of sensitization and natural desensitization to mosquito bites was described in the 1990s (Peng et al, 1966).

2- Large local reactions to mosquito bites (Skeeter Syndrome): Large local reactions are by far the most common type of allergic reactions to mosquito bites: 1- Large local reactions (termed Skeeter Syndrome) typically consist of an itchy or even painful area of redness, warmth, swelling and/or induration that ranges from a few $\mathrm{cm}$ to more than $10 \mathrm{~cm}$ in diameter. Large local reactions develop within hours of the bite, progress over 8 to 12 hours or more, and resolve within 3 to 10 days, 2- Large local reactions can involve the entire periorbital region and much of the face, or an entire extremity, especially in an infant or child. They can interfere with seeing, eating, drinking, or normal use of extremities. Severe large local reactions can be accompanied by low grade fever and malaise, 3- By inspection and palpation, it can be difficult to differentiate between allergic 
inflammation caused by mosquito bites and inflammation caused by secondary bacterial infection after scratching the bites. It is important to obtain a meticulous history of the time of onset of the red, warm swollen area at the site of a witnessed or likely mosquito bite in relationship to the time of the bite. Large local reactions typically begin within hours. Secondary bacterial infections typically begin within days and sometimes, develop an ecchymotic appearance or are associated with blisters, vesicles or bullae ( $\mathrm{Si}$ mons and Peng, 1999).

Systemic allergic reactions: Systemic allergic reactions to mosquito bites include papular or acute generalized urticaria, and rarely, asthma symptoms, anaphylaxis, serum sickness, or lymphadenopathy, hepatosplenomegaly, fever, and necrotic skin reactions at mosquito bite sites (Engler, 2001).

Epidemiology: People at increased risk of allergic reactions to mosquito bites include: 1- Those with a high level of exposure (e.g., civilian or military outdoor workers), 2- Infants and young children with low or absent natural immunity, 3- Immigrants or visitors to a geographic area where there are indigenous mosquitoes that they had not previously encountered and to which they have no natural immunity, and 4-Patients with primary or secondary immunodeficiency diseases (Peng et al, 2004a).

Clinical features and pathogenesis of large local reactions: The pathogenesis of Skeeter Syndrome was investigated in a study of five otherwise healthy young children age two to four years who developed large local reactions within hours of witnessed mosquito bites, and five age-matched control children who developed smaller typical reactions within hours of mosquito bites. All the children with the large local reactions had been diagnosed initially with bacterial cellulitis by primary care clinicians. All received systemic antibiotic treatment which was discontinued after a few days when the swelling was subsiding and the blood cultures performed at the time of initial presentation were reported as negative. In two of the children, radiographs were obtained to rule out osteomyelitis underlying the extensive soft tissue swelling at the site of a witnessed bite on an extremity (Naidu et al, 2008).

An ELISA was used to measure IgE \& IgG4 subclasses recognizing salivary gland allergens of the predominant indigenous mosquito Aedes vexans. The salivary allergens used in the ELISA were obtained by dissecting the salivary glands from the heads and thoraces of 370 laboratory-reared female A. vexans mosquitoes and dispersing them in $1 \mathrm{~mL}$ of diluent. In the children with Skeeter Syndrome, serum levels of IgE, $\mathrm{IgG} 1, \mathrm{IgG} 3$, \& $\mathrm{IgG} 4$ to $A$. vexans salivary gland allergens were significantly elevated as compared with the levels in control children. In Western blotting, sera from children with Skeeter Syndrome (but not from control children) reacted with 8 to $10 \mathrm{~A}$. vexans salivary gland allergens (Peng et al, 2004b). The IgE and IgG, especially IgG4 and IgG1, are involved in the development of Skeeter Syndrome. Serum mosquito salivary glandspecific IgG levels correlated significantly with the size of the immediate skin reaction to mosquito bites and with salivary glandspecific $\operatorname{IgE}$ levels. Specific $\operatorname{IgE}$ and $\operatorname{IgG}$ concentrations were significantly higher at the end of summer, as compared with levels at the end of the following winter after no exposure to mosquitoes had occurred for six months (Pauthner et al, 2016).

Natural history of large local reactions: In the absence of immune deficiency, the prognosis of Skeeter Syndrome appears to be favorable. As an example, children often continue to develop recurrent large local reactions to mosquito bites for several more summers, after which the reactions cease to occur. The time to resolution varies, however (Palosuo et al, 1997) and in some patients in northern latitudes (eg, Alaska, Canada, and the Nordic countries) this natural desensitization may take longer to develop because it depends on the frequency and intensity of the patient's exposure to mosquitoes, 
which is intermittent and limited in most cases by the short summer season. Additionally, it is reduced by efforts to avoid mosquitoes and prevent mosquito bites (Cohen and George, 2013).

In a subsequent study of sera from 402 children living in the same region of Canada where Skeeter Syndrome was originally described, levels of mosquito saliva-specific $\mathrm{IgE}$ and $\mathrm{IgG}$ correlated inversely with age, peaked at one to six months of age, and decreased after age five years (Peng et al, 2002). In this geographic region, only $18 \%$ of 1059 adult blood donors had demonstrable antibodies to mosquito salivary allergens, suggesting that in most adults, sensitization had been lost and natural desensitization had occurred.

Diagnosis: Skeeter Syndrome develops within hours of mosquito bites. Diagnosis is based on the time of onset of the reaction in relationship to a witnessed or likely mosquito bite, and on the physical finding of an itchy, red, warm swollen area at the site of the bite (Juckett, 2013). Testing for presence of antibodies to mosquito saliva is not practical because the only commercially available mosquito reagents are unstandardized whole body extracts that contain minimal mosquito salivary allergens but may contain irritant proteins (Peng and Simons, 1996). Obtaining pure mosquito salivary allergen for use in skin tests and in vitro tests by dissection of mosquito salivary glands, or by direct collection from living female mosquitoes, is labor-intensive and time-consuming. Such allergens are not commercially available. Mosquito bite challenge tests are contraindicated because of the risk of disease transmission through a "wild" mosquito bite, and the risk of causing another severe large local reaction in a susceptible patient (Wang et al, 2007).

Differential Diagnosis: The differential diagnosis of a large local reaction to a mosquito bite includes bacterial cellulitis and large local reactions to other insect bites and stings.
Bacterial cellulitis: The key information needed to facilitate the diagnosis of Skeeter Syndrome is the time elapsed (hours) between a witnessed mosquito bite, or exposure to mosquitoes and a likely mosquito bite, and the appearance of an itchy, red, warm, swollen, area at the bite site. A typical presentation of Skeeter Syndrome might involve a two-year-old child who presents with a history of playing in a park and being exposed to mosquitoes the previous evening, and then wakes up the next morning with an itchy, red, warm swelling of the entire periorbital region at the site of a mosquito bite. In contrast, bacterial cellulitis does not typically develop within hours of a mosquito bite. Instead, it appears several days later, after scratching and excoriation of the itchy area at the bite site and development of a secondary infection (Tay et al, 2014). On the other hand, Cellulitis is a common problem, caused by spreading bacterial inflammation of the skin, with redness, pain, and lymphangitis. Up to $40 \%$ of affected people have systemic illness. Gunderson and Martinello (2012) stated that erysipelas is a form of cellulitis with marked superficial inflammation, typically affecting the lower limbs and the face. The most common pathogens in adults are streptococci and Staphylococcus aureus. Cellulitis and erysipelas can result in local necrosis and abscess formation. Around a quarter of affected people have more than one episode of cellulitis within 3 years. They concluded that traditional view that cellulitis and erysipelas are primarily due to streptococcal species, with a smaller proportion due to $\mathrm{S}$. aureus, and also argue against the current distinction between cellulitis and erysipelas in terms of the relative proportion of infections due to $S$. aureus. Amer and Amer (2014) in Egypt reported that many rare cutaneous infections of the face, seemed irrelevant, because rare infections in certain parts of the world are common in others. For instance is cutaneous leishmaniasis (CL). Also, atypical mycobacterial infection was described in the medical 
literature since the mid-1950s. The development and introduction of a rapid radiometric mycobacterial detection system has advanced the field of mycobacteriology over the past 20 years. Mycobacterium tuberculosis from other mycobacteria and enabled the performance of antimicrobial susceptibility testing of mycobacteria. The increased frequency of atypical mycobacterial infection stems from advances in the diagnostic procedures concerning the infection paired with the prevalence of mycobacterial disease in immunocompromised patients infected with HIV.

Large local reactions to other insect bites and stings: Mosquito bites are painless, in contrast to painful bites from flies and other insects that cause large local reactions, and painful stings from bees, wasps, yellow jackets, yellow hornets, white-faced hornets, or fire ants that cause large local reactions.

Insect bites: One out of 17 deaths worldwide are due to a mosquito-transmitted arbovirus (yellow fever, dengue fever...etc.) protozoan (malaria...etc.) or helminthes (filariasis...etc.) diseases (Steen et al, 2004). However, insect bites in North America most commonly cause local inflammatory reactions that subside within a few hours and are no more than a nuisance (Vassallo et al, 2005). Generally speaking, most insect bites cause local inflammatory reactions that subside within a few hours. However, more severe local symptoms, transmission of a disease-causing pathogen, and systemic allergic reactions are also possible. Mosquito bites can cause varying degrees of local swelling, papular urticaria in children, and rare systemic allergic reactions, including anaphylaxis. Papular urticaria is a hypersensitivity reaction most often seen in children following mosquito and flea bites, although a variety of other bites have been implicated in smaller numbers of reports. Systemic allergic reactions can occur in response to the bites of mosquitoes, several types of bloodsucking flies, fleas, kissing bugs, lice, and ticks Morsy, 2012).
Insect stings when the venom is injected into the victim cause a variety of reactions, ranging from local irritation to life threatening anaphylaxis. Abdel Rahman et al. (2015) reported that hymenoptera are the third largest order of insects, comprising the sawflies, wasps, bees and ants. Worldwide, over 150,000 species are recognized, with many more remaining to be described. The name refers to the wings of the insects, but the original derivation is ambiguous. The Ancient Greek vuńv (hymen) for membrane provides a plausible etymology for the term because these insects have membranous wings. However, a key characteristic of this order is that the hind wings are connected to the fore wings by a series of hooks called hamuli. Thus, another plausible etymology involves, Hymen, the Ancient Greek god of marriage, as these insects have "married wings" in flight. Stinging insects and the medical risk associated with their venoms are complex topics, and presentation of information pertaining to them requires the use of technical terms. The most common reactions to these stings are transient pain and redness at the site lasting a few hours (local reaction), and exaggerated swelling lasting a few days (large local reaction). The most dangerous immediate reaction is anaphylaxis, which is potentially fatal.

Yellow jacket, wasps and Bee: Jin et al. (2010) reported that hyaluronidase is a minor yellow jacket venom allergen, and only $10 \%$ to $15 \%$ of patients with yellow jacket allergy are estimated to have IgE against the hyaluronidase protein. The peptide-specific cross-reactivity with Api $\mathrm{m} 2$ occurs in half of these sera. Component-resolved diagnosis with antigen 5 and phospholipase would detect virtually all patients with yellow jacket venom allergy. Witharana et al, (2015) in Sri Lanka conducted on patients presenting to Base Hospital Deniyaya with suspected bee and wasp stings from 2011 to 2013. Data were gathered using a questionnaire and specimens of offending insects collected for identification. They found that five speci- 
mens were available from those in anaphylactic shock (four Apis dorsata, one Ropalidia marginata). Vespa tropica stinging caused a characteristic skin lesion. They added that the risk factors included day-time outdoor activities, occupation (tea plantation wor-kers) and period of year. The latter may be due to pollen season when the insects are found in abundance. Only $4.6 \%$ of patients developed anaphylactic shock. Vespa tropi$c a$ stings led to a unique skin lesion.

Ant allergy: Ants have been called "chemical factories" (Morgan, 2008), since the venom of some ants may contain as many as 75 different components (Hoffman, 2010). Potiwat and Sitcharungsi (2015) in Thailand reported that hypersensitivity reactions caused by ant stings are increasingly recognized as an important cause of death by anaphylaxis. Only some species of ants (e.g. Solenopsis spp., Myrmecia spp., and Pachycondyla spp.) caused allergic reactions. Ant venom contains substances, including acids and alkaloids, that cause toxic reactions, and those from Solenopsis invicta or the imported fire ant have been widely studied. Piperidine alkaloids and low protein contents can cause local reactions (sterile pustules) and systemic reactions (anaphylaxis). Imported fire ant venoms are cross-reactive; for example, the Sol i 1 allergen from S. invicta has cross-reactivity with yellow jacket phospholipase. The Sol i 3 allergens is a member of the antigen 5 families that has amino acid sequence identity with vespid antigen 5 . They concluded that management of ant hypersensitivity can be divided into immediate (epinephrine, corticosteroids), symptomatic (antihistamines, bro-nchodilators), supportive (fluid resuscitation, oxygen therapy), and preventive

(resting avoidance and immunotherapy) treatments. Sanad et al. (2002) in Egypt reported that individuals from the suburb of Benha City and some adjacent villages were presented with various degrees of skin allergy; particularly children who spend the night (sleep) on the floor suffered generalized lymphadenopathy, with or without fever. The patients were treat-ed with carbolic acid (1:25). Besides, the oral anti-histamine (H1) and systemic antibiotics were indicated in the complicated cases. In the concrete houses of the patients, a huge number of the large ants (mainly Catagliphus bicolar) were found moving here and there, particularly in animal house included indoors. Spreading or burning dried leaves of camphor tree proved to an effective repellent for the ants.

Scorpion sting: Scorpion stings pose a considerable threat for public health in many regions of the world, especially in less- developed countries of tropics and subtropics. A list of high risk areas includes: Saharan Africa, Sahelian Africa, South Africa, the Near and Middle East, South India, Mexico, and South Latin America, to the east of the Andes. Cumulatively, a total of 2.3 billion persons are exposed to scorpion stings. Furthermore, scorpions inhibit also the south areas of the Unites States and Southern Europe (Kluz-Zawadzka et al, 2014). An estimated 1.2 million persons are stung by scorpion per year. Of them, ca 3,250 persons $(0.27 \%)$ die. In fact, an actual incidence is unknown as the majority of scorpion stings occur in less-developed countries, in remote rural areas, jungles and deserts, remaining unreported (Chippaux and Goyffon, 2008). Molaee et al. (2014) in Iran reported that the scorpion activity in Dezful County is a climatological-dependent phenomenon.

In Egypt, not less than 100 authors dealt with scorpion. Farghly and Ali (1999) and Meki et al. (2003) stated that the scorpion as a real environmental health problem in Upper Egypt mainly among the pre-school and school aged children. The major fatty acids in the he-patopancreas were oleic, linoleic and Palmitic acids (Abul-Fadl, 1960). Engelmann and Hallof (2004) considered the scorpions as one of the medical occupational problem in Egypt's early times. They were responsible for precautionary measures against epidemics and for curing snake and scorpion bites. They created the first medical 
papyri and established the legal foundation for the medical care of the inhabitants of Egypt by royal order. Morsy (2009) in Toshka District reported about 18 species of scorpions Fatani et al. (2010) reported that scorpion was a real problem and compared between the protective effects of Saudi and Egyptian anti-venoms. The scorpion venomous species cause severe systemic reactions, lymphadenitis, twitching, muscle spasm and convulsions. Besides, the patients may die of respiratory paralysis with pulmonary edema within 2 to 3 hours after being sting (Efrati, 1949). Shoukry and

Fetaih et al. (2013) injected experimental mice with scorpion venom. They found that the most obvious changes in the liver were acute cellular swelling, hydropic degeneration, congestion of central veins and portal blood vessels. Besides, extramedullary hematopoiesis and invaginations in nuclei of hepatic cells, with formation of intranuclear cytoplasmic inclusions were observed. Mohammad et al. (2014) evaluated demographic and clinical characteristics as well as outcomes in referred children to Assiut University Children Hospital during the year 2012 with a history of scorpion sting. They concluded that more than half of stung children had a severe clinical presentation and about one fifth died and that aggressive treatment regimens were recommended for such patients to improve the outcome. Ahmed et al. (2015) reported that endocrinological changes were common in all children with scorpion envenomation and more obvious in cases of severe envenomation. The released mediators may account for several inflammatory manifestations such as pulmonary edema, myocardial failure, systemic inflammatory response syndrome and multiple organ failure. The use of insulin is recommended in cases of severe envenomation to improve the outcome. On the other hand, Chen et al. (2012) in China demonstrated that peptide $\mathrm{Kn} 2-7$ of scorpion venom could inhibit HIV-1 by direct interaction with viral particle and may become a promising candi- date compound for further development of microbicide against HIV-1. Also, Ding et al. (2015) in China reported that BmKTT-2 was the first Kunitz-type human plasmin inhibitor from scorpion venom, providing novel insights into drug developments targeting human plasmin protease.

Spider bite: Generally, Spiders are found worldwide on every continent except for Antarctica, and have become established in nearly every habitat with the exceptions of air and sea colonization. As of November 2015 , at least 45,700 spider species, \& 113 families were recorded. However, there has been dissension within the scientific community as to how all these families should be classified, as evidenced by the over 20 different classifications that have been proposed since 1900 (Foelix, 1996), While the venom of a few species is dangerous to humans, scientists are now researching the use of spider venom in medicine and as nonpolluting pesticides. Spider silk provides a combination of lightness, strength and elasticity that is superior to that of synthetic materials, and spider silk genes have been inserted into mammals and plants to see if these can be used as silk factories. Wide range of behaviors, spiders have become common symbols in art and mythology symbolizing various combinations of patience, cruelty and creative powers. Fear of spiders is called arachnophobia.

All possible symptoms include: itching or rash, pain around the area of the bite, muscle pain or cramping, blister that's red or purple in color, sweating, difficulty breathing, headache, nausea and vomiting, fever, chills, anxiety or restlessness, swollen lymph glands, and high blood pressure. Bites take longer to heal than insect bites, and they may affect skin tissues. It's important to keep bite clean to reduce infection risk.

Spiders have been incriminated as causes of human suffering for centuries, but few species worldwide cause medically significant envenomation dangerous to people (Vetter and Isbisterm, 2008). Spiders only 
bite man in self-defense, and few produce worse effects than a mosquito bite or beesting. Widow spiders occur worldwide and cause latrodectism, characterized by pain (local and generalized) associated with nonspecific systemic effects, diaphoresis, and less commonly other autonomic and neurological effects (Hannum and Miller, 2008). Recluse spiders are distributed mostly in the tropical and subtropical Western Hemisphere and can cause severe skin lesions and rarely systemic effects; most bites are unremarkable. High risky spiders in South America (armed spiders) and Australia (funnelweb spiders) cause rare but severe envenomation requiring medical intervention and sometimes antivenom. Most other spiders involved in verified bites cause minor, transient effects (Vetter and Barger, 2002). Funnel web spiders' defensive tactics include fang display and their venom, although they rarely inject much, has resulted in 13 known human deaths over 50 years. Australian funnel-web spiders are generally considered the most dangerous spiders in the world, with envenomations from the Sydney funnel-web spider Atrax robustus resulting in at least 14 human fatalities prior to the introduction of an effective anti-venom in 1980 (Pineda et al, 2012), though this claim has also been attributed to the Brazilian wandering spider, due to much more frequent accidents. Richardson et al. (2006) reported that the proteomes of the venoms of the Brazilian wandering (armed) spiders Phoneutria nigriven-ter, Ph. reidyi, \& Ph. keyserlingi were compared using two-dimensional gel electrophoresis. Venom components were fractionated using a combination of preparative reverse phase HPLC on Vydac C4, analytical RP-HPLC on Vydac C8 and C18 and cation exchange FPLC on Resource S at pH $6.1 \& 4.7$, or anion exchange HPLC on Synchropak AX-300 at $\mathrm{pH}$ 8.6. Amino acid sequences of native and S-pyridyl-ethylated proteins and peptides derived from them by enzymatic digestion and chemical cleavages were determined using a Shimadzu PPSQ-21(A) auto- mated protein sequencer, and by MS/ MS collision induced dissociations

There were about 100 reliably reported deaths from spider bites in the 20th century (Williamson et al, 1996) compared to about 1,500 from jellyfish stings (Nishioka, 2001). Many alleged cases of spider bites may represent false diagnoses (Isbister, 2001), made it more difficult to check the effectiveness of treatments for genuine bites (Diaz, 2004).

Antihistamines: In randomized doubleblind placebo-controlled trials of patients bitten by mosquitoes in laboratory and field settings, prophylactic administration of a non-impairing, non-sedating H1-antihistamine, (e.g., Cetirizine, Desloratadine, Fexofenadine, Levocetirizine, or Loratadine ) relieved itching in the early-phase allergic reaction and reduced the late-phase reactions (redness, swelling, and induration). H1-antihistamines are the only class of pharmacologic agents that has been studied prospectively in prevention or treatment of large local reactions to insect stings or bites (Karppinen et al, 2006). Patients with a nonimpairing, non-sedating antihistamine be taken on a regular daily basis when mosquito exposure was inevitable, which must be used to treat itching, redness, and swelling that occur at mosquito bite sites.

Short- or long-term safety of cetirizine, desloratadine, fexofenadine, levocetirizine, and loratadine was proved in randomized control trials, even in young children. Safety of impairing, sedating $\mathrm{H} 1$-antihistam-ines such as diphenhydramine and chlorpheniramine was not well studied in controlled trials (Simons and Simons, 2011).

Glucocorticoids: Glucocorticoids are used to treat the allergic inflammation associated with large local reactions to stinging insects. This approach extrapolated to the treatment of large local reactions to mosquito bites (Golden et al, 2011).

Arthropods irritation: To reduce irritation, a moderately potent topical glucocorticoid cream with a good benefit-to-risk ratio, for example, mometasone cream $0.1 \%$, applied 
twice daily for 5 to 10 days. For severe large local reactions, distressing and/or interfere with normal vision, ingestion of liquid or food, or ambulation, an oral glucocorticoid as prednisone $1 \mathrm{mg} / \mathrm{kg}$ to a maximum of 50 mg may be given for 5 to 7 days. Many topical lotions and creams with local anesthetics and/or H1-antihistamines as diphenhydramine or doxepin are available for application to affected mosquito bite sites; however, these interventions are not recommended because topical application of these medications can lead to systemic absorption and contact hypersensitivity. Antibiotic is not indicated for Skeeter Syndrome developed within hours, because bacterial infection would develop within this brief time.

\section{Nurse Management Guidelines}

Allergic response: 1- Difficulty/noisy breathing, 2- Swelling of lips, tongue, face, eyes, 3- Swelling/tightness in throat, 4- Difficulty talking and/or hoarse voice, 5- Difficulty swallowing, 6- Pain at bite/sting site, 7Vomiting, 8- Abdominal pain, 9- Wheeze or cough, 10- Erythema or urticarial rash, 11History of allergy to an insect bite or sting.

Signs of envenomation/ neurotoxic paralysis: 1- Eye lids drop, 2- Decrease/eye paralysis, 3- Leg weakness, 4-Abnormal respiration (Emerging Care Institute, 2016).

Management:1- Remove insect and stinger if still attached to skin, 2- When removing stingers, use a sideways scraping motion to avoid further envenomation, 3- Inspect patient's clothing and remove any other insects and stingers, 4- Apply a cold pack at $20 \mathrm{mi}-$ nute on / off intervals for pain relief and to reduce swelling, 5- Provide further analgesia as required according to pain scale, 6Administration of tetanus toxoid as immunization history, 7- Document assessment findings, interventions and outcomes.

\section{Conclusion and Recommendations}

People bitten by mosquitoes become sensitized to salivary allergens, but few develop mosquito allergy, most commonly large local reactions to mosquito bites. Systemic reactions, including anaphylaxis, are rare.
Skeeter Syndrome is caused by an allergic reaction to mosquito saliva. These itchy, red, warm swellings typically develop within hours of bites on the face or extremities, and resolve with or without treatment over 3 to 10 days. Skeeter Syndrome is commonest in young children who have not yet developed natural immunity to mosquitoes, and may be accompanied by low-grade fever and malaise.

Skeeter Syndrome is diagnosis is clinically. The key information needed is the time elapsed, measured in hours (not days) between a witnessed mosquito bite or exposure to mosquitoes and a likely mosquito bite, and the appearance of an itchy, red, warm, swollen area at the bite site. Prompt recognition and appropriate treatment helps avoid unnecessary diagnostic procedures and unnecessary antibiotic treatment. Skeeter Syndrome is frequently misdiagnosed as bacterial cellulitis; however, Skeeter Syndrome typically develops within hours of a bite, and bacterial cellulitis typically develops a few days later, after bite sites have been scratched and excoriated. Clinical diagnosis of allergic reactions to mosquito bites is difficult because of lack of availability of highquality standardized allergens (mosquito salivary gland or saliva extracts) for skin tests or measurement of serum mosquito salivaspecific IgE levels. In healthy children, large local reactions (Skeeter Syndrome) typically cease to occur within a few years.

Environmental measures to limit mosquito breeding habitats and repellents to prevent bites are the primary means of preventing large local reactions in patients with a history of past reactions. If mosquito exposure is predictable or inevitable, recommends a non-impairing, non-sedating $\mathrm{H} 1$-antihistamine, taken before spending time outdoors (Grade 1B). Treatment of large local reactions to mosquito bites is symptomatic: $\mathrm{Pa}-$ tients with bothersome itching; recommended an oral non-impairing, non-sedating H1antihistami-ne (Grade 1B). Cetirizine, at age appropriate doses for patients with swelling 
and induration several centimeters in diameter, a topical glucocorticoid cream suggest (Grade 2C), typically use mometasone $0.1 \%$, twice daily for 5-10 days. Patients with extensive periorbital swelling interferes with vision, lip swelling or with liquid or food ingestion, or swelling of a limb that interferes with ambulation, a short course of an oral glucocorticoid as prednisone $(1 \mathrm{mg} / \mathrm{kg}$ up-to $50 \mathrm{mg}$ ) once daily for 5-7 days (Grade 2C).

\section{References}

Abdel-Rahman, RZ, Mohamad, HM, Morsy, ATA, Morsy, TA, 2015: Allergic reactions caused by venom of hymenopterous stinging insects and the role of health care workers. J. Egypt. Soc. Parasitol. 45, 2:403-12.

Ahmed, AE, Abdel-Baseer, KA, Saad, K, Hassan, AF, El-Houfey, AA, 2015: Endocrinological and biochemical changes of scorpionism in children in Upper Egypt. Ther. Adv. Endocrinol. Metab. 6, 5:210-6

Amer, M, Amer, A, 2014: Rarely seen infections. Clin. Dermatol. 32, 6:744-51.

Chen, Y, Cao, L, Zhong, M, Zhang, Y, Han, C, et al, 2012: Anti-HIV-1 activity of a new scorpion venom peptide derivative Kn2-7. PLoS One 7(4):e34947. doi: 10.1371/journal.

Chippaux, JP, Goyffon, M, 2008: Epidemiology of scorpionism: A global appraisal, http. dx. doi.org/ 10. 1016/j.actatropica.2008.05.021

Cohen, B, Hebert, AA, 2011: Assault of arthropods, which product should you recommend to prevent insect bites? At: www.aapnews.org.

Cohen, A, George, O, 2013: Animal models of nicotine exposure: Relevance to second-hand smoking, electronic cigarette use, and compulsive smoking. Front Psychiatry. 4:41. Published online Doi: 10.3389/ fpsyt. 00041

Diaz, JH, 2004: The Global epidemiology, syndromic classification, management, and prevention of spider bites. Am. J. Trop. Med. Hyg. 71, 2:239-50.

Ding, L, Wang, X, Liu, H, San, M, Xu, Y, et al, 2015: A new Kunitz-type plasmin inhibitor from scorpion venom. Toxicon 106:7-13.

Efrati, P, 1949: Poisoning by scorpion sting in Israel. Amer. J. Trop. Med. 29: 249-57.

Engelmann, H, Hallof, J, 2004: Studies on the development of medical occupational image in Egypt's early times. Med. Ges. Gesch. 23:9-42.

Engler, RJ, 2001: Mosquito bite pathogenesis in necrotic skin reactors. Curr. Opin. Allergy
Clin. Immunol. 1:349-54.

Farghly, WM, Ali, FA, 1999: A clinical and neurophysiological study of scorpion envenomation in Assiut, Upper Egypt. Acta Paediatr. 88, 3:290-4

Fatani, AJ, Ahmed, AA, Abd-el-Halim, RM, Abdoon, NA, Darweesh, AQ, 2010: Comparative study between the protective effects of Saudi and Egyptian anti-venoms, alone or in combination with ion channel modulators, against deleterious actions of Leiu-rus quinquestriatus scorpion ven-om. Toxicon. 55, 4:773-86

Fetaih, HA, Shoukry, NM, Soliman, BA, Mohallal, ME, Khaled, HS, 2013: Histopathological changes in liver of mice after experimental envenomation with Androctonus amoreuxi scorpion venom. J. Egypt. Soc. Parasitol. 43, 2:44756.

Foelix, RF, 1996: Biology of Spiders. 198 Madison Ave. NY, New York, 10016: Oxford University Press.

Golden, DB, Moffitt, J, Nicklas, RA, et al, 2011: Stinging insect hypersensitivity: a practice parameter update 2011. J. Allergy Clin. Immunol. 127:852-9.

Gunderson, CG, Martinello, RA, 2012: A systematic review of bacteremias in cellulitis and erysipelas. J. Infect. 64, 2:148-55.

Hannum, C, Miller, DM, 2008: Widow Spiders: Department of Entomology, Virginia Tech. Hoffman, DR, 2010: Ant venoms. Curr. Opin. Allergy Clini. Immunol. 10, 4:342-6

Isbister, GK, 2001: Spider mythology across the world. West. J. Med. 175, 4:86-7.

Jin, C, Focke, M, Léonard, R, Jarisch, R, Altmann, F, et al, 2010: Reassessing the role of hyaluronidase in yellow jacket venom allergy. $\mathrm{J}$. Allergy Clin. Immunol. 125, 1:184-90.e1.

Juckett, G, 2013: Arthropod bites. Am. Fam. Physician. 88, 12:841-7

Karppinen, A, Brummer-Korvenkontio, H, Petman, L, et al, 2006: Levocetirizine for treatment of immediate and delayed mosquito bite reactions. Acta Derm. Venereol. 86:329-34. Katz, TM, Miller, JH, Hebert, AA, 2008: Insect repellents: historical perspectives \& new developments. J. Am. Acad. Dermatol. 58:865-8. Kluz-Zawadzka, J, Hartman-Ksycińskam A, Lewandowski, B, 2014: Emergent management of scorpion sting. Przegl. Epidem. 68, 4:685-8. Meki, AR, Mohamed, ZM, Mohey-El-deen, H M, 2003: Significance of assessment of serum cardiac troponin I and interleukin-8 in scorpion 
envenomed children. Toxicon. 41, 2:129-37.

Mellanby, K, 1946: Man's reaction to mosquito bites. Nature 158:554.

Mohammad, IL, Elsayh, KI, Mohammad, H A, Saad, K, Zahran, AM, et al, 2014: Clinical characteristics and outcome of children stung by scorpion. Eur. J. Pediatr. 173, 6:815-8.

Molaee, SM, Ahmadi, KA, Vazirianzadeh, B, Moravvej, SA, 2014: A climatological study of scorpion sting incidence from 2007 to 2011 in the Dezful area of southwestern Iran, using a time series model. J. Insect Sci. 1, 14:151-9. Morgan, DE, 2008: Chemical sorcery for sociality: exocrine secretions of ants (Hymenoptera: Formicidae) Myrmecological News 11:79-90.

Morsy, TA, 2012: Insect bites and what is eating you? J. Egypt. Soc. Parasitol. 42, 2:291-308. Naidu, DK, Ghurani, R, Emerick Salas, R, Mannari, RJ, et al, 2008: Osteomyelitis of the mandibular symphysis caused by brown recluse spider bite. Eplasty 8: e45. Published online.

Nishioka, $S$ de A, 2001: Misdiagnosis of brown recluse spider bite. West. J. Med. 174, 4:240.

Palosuo, K, Brummer-Korvenkontio, H, Mikkola, J, et al, 1997: Seasonal increase in human $\mathrm{IgE}$ and IgG4 antisaliva antibodies to Aedes mosquito bites. Int. Arch. Allergy Immunol. 114:367-72.

Pauthner, M, Yeung, J, Ullman, C, Bakker, J, Wurch, T, et al, 2016: Antibody engineering \& therapeutics, the annual meeting of the antibody society December 7-10, 2015, San Diego, CA, USA. MAbs. 8, 3: 617-52: Published online.

Peng, Z, Simons, F, 1996: Comparison of proteins, IgE, \& IgG binding antigens, and skin reactivity in commercial and laboratory-made mosquito extracts. Ann. Allergy Asthma Immunol. 77:371-4.

Peng, Z, Simons, FE, 2007: Advances in mosquito allergy. Curr. Opin. Allergy Clin. Immunol. 7:350-8.

Peng, Z, Yang, M, Simons, F, 1966: Immunologic mechanisms in mosquito allergy: correlation of skin reactions with specific IgE \& IgG antibodies \& lymphocyte proliferation response to mosquito antigens. Ann. Allergy Asthma Immunol. 77:328-41.

Peng Z, Rasic N, Liu Y, Simons F, 2002: Mosquito saliva-specific IgE \& IgG antibodies in 1059 blood donors. J. Allergy Clin. Immunol. 110:816-201

Peng, Z, Ho, MK, Li, C, Simons, FE, 2004a: Evidence for natural desensitization to mosquito salivary allergens: mosquito saliva specific $\operatorname{IgE}$ and IgG levels in children. Ann. Allergy Asthma Immunol. 93, 6:553-6.

Peng Z, Beckett AN, Engler RJ, et al, 2004b: Immune responses to mosquito saliva in 14 individuals with acute systemic allergic reactions to mosquito bites. J. Allergy Clin. Immunol. 114: 1189-92.

Potiwat, R, Sitcharungsi, R, 2015: Ant allergens and hypersensitivity reactions in response to ant stings. Asian Pac. J. Allergy Immunol. 33, 4:267-75.

Reunala, T, Brummer-Korvenkontio, H, Räsänen, L, et al, 1994: Passive transfer of cutaneous mosquito-bite hypersensitivity by $\operatorname{IgE}$ anti-saliva antibodies. J. Allergy Clin. Immunol. 94:902-9.

Richardson, M, Pimenta, AM, Bemquerer, M P, et al, 2006: Co-mparison of the partial proteomes of the venoms of Brazilian spiders of the genus Phoneutria. Comp. Biochem. Physiol. C Toxicol. Pharmacol. 142, 3/4:173-87.

Sanad, EM, Helmy, AZ, Morsy, TA, 2002: Ant allergy in Benha District Qualyobia G., Egypt. J. Egypt. Soc. Parasitol. 32, 3:901-6.

Shoukry, NM, Morsy, TA, 2009: Arthropod borne diseases at Toshka, Upper Egypt. World J. Zool. 6, 2:126-33

Simons, FE, Peng, Z, 1999: Skeeter syndrome. J. Allergy Clin. Immunol. 104:705-10.

Simons, FE, Simons, KJ, 2011: Histamine and H1-antihistamines: celebrating a century of progress. J. Allergy Clin. Immunol.128:1139-42.

Steen, CJ, Carbonaro, PA, Schwartz, RA, 2004: Arthropods in dermatology. J. Am. Acad. Dermatol. 50, 6:819-44.

Tay, EY, Thirumoorthy, T, Pang, SM, et al, 2014: Clinical outcomes of bacteraemia in cellulitis of the leg. Clin. Exp. Dermatol. 39, 6:683-8 Vassallo, C, Passamonti, F, Cananzi, R, et al, 2005: Exaggerated insect bite-like reaction in patients affected by oncohaematological diseases. Acta. Derm. Venereol. 85:76-80.

Vetter, RS, Barger, DK, 2002: An infestation of 2,055 brown recluse spiders (Araneae: Sicariidae) and no envenomations in a Kansas home: implications for bite diagnoses in nonendemic areas. J. Med. Entomol. 39, 6: 948-51.

Vetter, RS, Isbisterm GK, 2008: Medical aspects of spider bites. Ann. Rev. Entomol. 53: 409-29. 